\title{
Volume !
}

VOLUME! La revue des musiques populaires

$18: 2 \mid 2021$

Experts - Non Experts

\section{Alessandro BRATUS, Mediatization in Popular Music Recorded Artifacts: Performance on Record and on Screen}

Catherine Rudent

\section{OpenEdition}

\section{Journals}

Édition électronique

URL : https://journals.openedition.org/volume/9553

DOI : 10.4000/volume.9553

ISSN : 1950-568X

Éditeur

Association Mélanie Seteun

Édition imprimée

Date de publication : 15 décembre 2021

Pagination : 179-184

ISBN : 9782913169678

ISSN : 1634-5495

Référence électronique

Catherine Rudent, "Alessandro Bratus, Mediatization in Popular Music Recorded Artifacts: Performance on Record and on Screen », Volume! [En ligne], 18 : 2 | 2021, mis en ligne le 15 décembre 2021, consulté le 30 juillet 2022. URL : http://journals.openedition.org/volume/9553 ; DOI : https://doi.org/10.4000/ volume.9553 
plutôt que de recourir aux voix des femmes qu'il avait mobilisées pour l'occasion. La conception de cet album est l'occasion de pointer de nombreuses négociations entre les différents protagonistes de l'album à travers notamment le récit qu'en fait JeanPaul Romann, le directeur artistique qui relate le travail de «médiation relationnelle et technique » qui a permis l'inscription d'un retour aux sources voulu par les musiciens qui néanmoins n'en partageaient pas tous la même vision, "dans un album fait pour le marché international» (258).

Les voix de ce directeur, celles des musiciens et musiciennes, ingénieurs du son, spectateurs de festival en France et au Mali forment la polyphonie de ce livre qui décrit ainsi la mondialisation des musiques touarègues à partir de multiples perspectives. Cette attention aux acteurs est liée au refus des généralités sur la mondialisation des productions musicales auxquelles l'auteur a préféré une convaincante ethnographie détaillée et itinérante des différentes opérations faisant aboutir une musique touarègue. Elle peut ainsi éclairer la fabrique relationnelle de cette musique au gré de circulations artistiques, de dynamiques de normalisation culturelle, et de rencontres et confrontations des conceptions sonores et des imaginaires du désert.

Bibliographie

Doyle Peter (2005), Echo and Reverb, Fabricating Space in Popular Music Recording 1900-1960, Middletown, Wesleyan University Press.

Gabry-Thienpont Séverine (2017), “ Du Caire à Nantes. Parcours et reformulations du zār, de ses musiques et de ses acteurs ", Cahiers d'ethnomusicologie, n 30 , p. 137-153.
Le Menestrel Sara (coord.), Christophe Apprill, Kali Argyriadis, Julien Mallet, Nicolas Puig, Guillaume Samson, Gabriel Segré (2012), Des Vies en musique. Parcours d'artiste, mobilités, transformations, Paris, Éditions Hermann.

\section{Alessandro Bratus, Mediatization in Popular Music Recorded Artifacts : Performance on Record and on Screen, Lanham, Lexington Books, 2019}

\section{Par Catherine Rudent}

Mediatization in Popular Music Recorded Artifacts : Performance on Record and on Screen, d'Alessandro Bratus traite un sujet déjà bien défriché en popular music studies : la relation entre musique live et musique médiatisée. Il l'aborde en s'intéressant à deux familles d'objets, les films de concerts et les albums posthumes, objets ayant eux aussi suscité une bibliographie nourrie.

Avec de telles ambitions générales, une des premières qualités qui frappent dans l'ouvrage est justement sa maîtrise de 
la bibliographie sur les principales thématiques abordées. L'auteur semble avoir une aptitude exceptionnelle à découvrir, assimiler et citer toutes sortes de travaux, non seulement les références incontournables sur les questions qu'il se pose, mais aussi des ouvrages de sciences sociales ou de philosophie plus généraux qu'il utilise pour opérer des mises en perspective inattendues. En outre, ce vaste panorama de références est équilibré, en ce qu'il combine des apports de plusieurs contextes intellectuels, ajoutant aux habituelles références anglophones d'importants travaux italiens - Bratus est professeur à l'université de Pavie - et français. Un autre trait frappant de ce paysage de références large et maîtrisé est l'abondance de travaux signés par des femmes, certains notoires, d'autres beaucoup moins.

La largeur de vue apparaît donc d'emblée comme une des caractéristiques du livre. Cela se vérifie si l'on considère son ampleur théorique. L'auteur y développe non pas une thèse principale mais plusieurs, centrées chacune sur différents concepts et dont voici une présentation succincte. Tout d'abord, le média (enregistrement, film) construit des sens, des interprétations, pour les musiques qu'il présente. Il vise entre autres à les situer selon des « coordonnées » dans le « système des genres de musiques populaires ». Mais les films musicaux et enregistrements posthumes construisent aussi une identité de «leur» public, incitant l'auditeur ou spectateur à se positionner par rapport à ce public visé.

On peut observer le processus de ces constructions si l'on analyse les objets médiatisés (performative objects : les films, les albums) en les comparant avec le concept préexistant à la performance (performative concept) et avec sa mise en œuvre lors de l'enregistrement

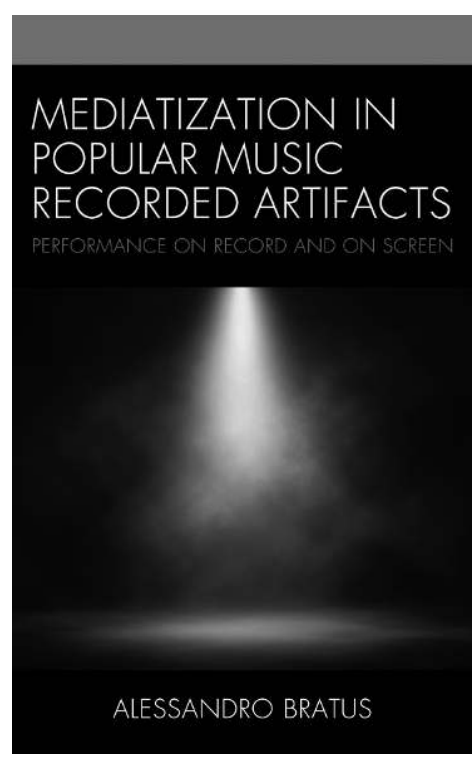

(performative event). Il s'avère alors que les albums posthumes relient live et média plutôt qu'ils ne les opposent : ils montrent bien comment l'aspect « vivant» est indépendant de la vie physique de l'artiste enregistré. Dès lors, il apparaît que les films de concerts, eux aussi, construisent du live plutôt qu'ils ne le retranscrivent. Cette construction du live se complète seulement lors de l'authentification de l'objet médiatisé par l'auditeur ou le spectateur. C'est à lui de choisir d'y croire (will to believe, concept repris du philosophe et psychologue William James) par un «saut» (leap) qui ne peut venir que de lui, même si Bratus montre comment un tel saut est visé par différentes stratégies des objets médiatiques analysés.

De manière récurrente, cette ligne de réflexions s'appuie aussi sur la notion d'auctorialité : celle-ci vient renforcer ou affaiblir, selon les cas, la dynamique d'authentification de l'objet médiatique considéré. Il en résulte qu'identité (des artistes filmés et enregistrés) et authenticité sont évolutives 
au fil des contextes de leur publication : ainsi les enregistrements médiatisés en disent plus sur leur propre contexte que sur l'évènement qu'ils sont censés présenter, parfois des décennies après son enregistrement.

Ces différentes thèses apparaissent de manière récurrente au fil d'un propos dense et organisé de manière non linéaire. Malgré une introduction ambitieuse et un premier chapitre qui se veut méthodologique, la saisie de la pensée de l'auteur ne se fait qu'au fil des chapitres et c'est seulement après une centaine de pages environ qu'une synthèse en devient possible pour le lecteur.

Cette densité, qui frôle par endroits le désordre, est heureusement contenue par un chapitrage limpide. Chaque chapitre est centré sur un ou plusieurs des éléments du corpus et en propose une analyse approfondie, généralement convaincante, quelquefois impressionnante. Ainsi, si l'on ne peut se reposer sur l'auteur pour nous guider clairement dans les circonvolutions de sa réflexion théorique, l'on peut en quelque sorte " choisir de croire " à la productivité de ses propositions, grâce à ses analyses. Le lecteur fait l'expérience d'un « saut » épistémologique similaire au will to believe évoqué tout au long du livre, à moins qu'il ne choisisse de rester en dehors.

Mediatization in Popular Music s'articule autour d'une dizaine d'études de cas : «Volunteers » de Jefferson Airplane (chapitre I) - la version studio et deux versions live; la production d'albums posthumes de Jimi Hendrix (chapitre 2); celle de Tupac Shakur (chapitre 3); la collection des American de Johnny Cash, avant et après son décès (chapitre 4); deux films de "concerts " sans public, celui de Korn (The Encounter) et celui du Pink Floyd (Live at Pompeii) dans le chapitre 5 ; trois films de concerts d'artistes
d'EDM - The Prodigy, The Chemical Brothers, Fatboy Slim - comparés dans le chapitre 6 ; enfin, la conclusion s'appuie sur des analyses de $T h i s$ is $i t$, film consécutif au décès de Michael Jackson.

Reprenant chaque fois l'ensemble des cadrages théoriques susmentionnés, ces analyses aboutissent à des résultats agréablement diversifiés.

Le chapitre I dégage les spécificités de trois médiums considérés pour une même chanson, l'album studio, la scène filmée (à Woodstock, telle qu'elle apparaît dans le film de Michael Wadleigh) et la télévision (au Dick Cavett Show, I9 août I969). La comparaison est approfondie et multidirectionnelle. Elle établit par exemple que le montage plus serré du film indique une patte artistique du réalisateur, là où la télévision se veut une fenêtre sur la réalité (44). Des différences structurelles entre les trois versions de la chanson (fonction de l'introduction, développement de sections improvisées) sont dégagées et mises en rapport avec chaque évènement (event) considéré. Le chapitre 2 est à mon sens le plus impressionnant et le plus convaincant de l'ouvrage. Traitant des différents enregistrements posthumes de Jimi Hendrix, il montre comment, selon les versions de tel ou tel titre, le producteur (qui varie) a mis en avant l'image d'un Hendrix expérimentateur et compositeur de studio ou celle d'un performeur exceptionnel et virtuose de la guitare. Appuyé sur des schémas formels comparatifs nombreux et lisibles, il permet aussi à l'auteur de montrer comment, au fil des décennies de sa "carrière posthume", la vision de Hendrix par ses producteurs se rétrécit autour de la seconde image, faisant état d'une rigidification passéiste des personas possibles pour l'artiste et inscrivant ces albums bien 
plus dans leur contemporanéité que dans le moment passé qu'ils sont supposés prolonger. Similaire dans ses intentions, l'analyse de la carrière posthume de Tupac (chapitre 3) met bien en évidence un cas d'auctorialité mal reçue : quand Eminem travaille à sa manière des pistes vocales inédites de Tupac dans Loyal to the Game, en 2004, il ne peut emporter l'adhésion du public - son will to believe - en raison d'interventions trop apparentes de son propre style et de l'absence de toute coopération effective entre les deux rappeurs dans le passé. Le chapitre 4 montre au contraire comment l'identité de Johnny Cash à travers American Recordings et la suite des American - les deux derniers sont posthumes - semble capable de tout intégrer, ce que Bratus appelle joliment une «identité expansive». La mutation du chanteur de country passé de mode en un repreneur admiré et éclectique, capable de s'approprier les plus contemporains des courants de rock américain, s'est opérée de son vivant; le mouvement a ainsi pu être poursuivi après sa mort sans rupture de l'effet de vivant (liveness) et sans réticence de la part d'un public lui aussi élargi, qui n'a cessé $\mathrm{d}^{\prime}$ " authentifier » ces albums. Le chanteur a pu finalement quitter sa persona de «contradiction vivante " au profit d'une image d'ampleur quasi illimitée dans sa capacité d'inclusion.

En analysant d'abord The Encounter (Korn, 20IO), puis un de ses principaux modèles, Live at Pompeii (Pink Floyd, I972), le chapitre 5 quitte la question des albums posthumes pour entrer dans une séquence de trois chapitres consacrés à des films de concerts - tous atypiques : dans le chapitre 5 , ce sont des concerts sans public, dans le sixième, des concerts dans une certaine mesure sans musiciens, enfin dans la conclusion, This is
Le chapitre 5 est l'occasion pour l'auteur de théoriser la médiatisation comme construction d'un évènement, au lieu d'être sa reproduction. Ceci l'amène à développer quelques réflexions sur le concept d'évènement qui, pour être aux limites du compréhensible, n'en sont pas moins suggestives - aboutissant à une citation d'Alain Badiou, étrangement laissée en français - avec quelques coquilles excusables (187). Le sixième et dernier chapitre est aux prises avec un autre corpus paradoxal : celui des concerts d'EDM - il est en fait centré sur trois des artistes réputés avoir initié le big beat en Angleterre : The Chemical Brothers, The Prodigy et Fatboy Slim. L'auteur considère ici que la notion de concert a un sens inhabituel dans le cadre de l'EDM (191) et surtout que la « performativité " y est particulière, étant donné l'implication moindre du corps du musicien, le rôle plus prégnant joué par les machines et l'importance de sons préexistants au concert (212-213). Enfin la conclusion est remplacée par une «Coda » présentant un cas-limite complémentaire, celui de This is $i$. Bratus considère Michael Jackson comme un « artiste audiovisuel » plutôt que seulement un musicien : ses chansons sont, tout au long de sa carrière, des œuvres où la danse et les images enregistrées sont aussi importantes que la musique et la vocalité. Il souligne comment This is it, alors même qu'il se présente comme une trace documentaire des préparatifs d'un spectacle jamais donné ( $a$ would-be concert film "), obéit néanmoins à une stratégie narrative et communicationnelle. Le film juxtapose, pour une même chanson, des enregistrements issus de nombreux moments disparates des répétitions et use d'autres procédés de «fragmentation », telle la division de l'écran, autant d'artifices qui demandent au spectateur une participation 
active à « l'effacement des discontinuités ». Mais, en progressant vers sa fin, le film évolue vers une attitude plus documentaire et réaliste, ce qui est évident pour les deux dernières chansons, «Billie Jean » et «Man in the Mirror ", qui « témoignent d'une unique performance, avec aussi peu de post-production et de montage que possible». Il permet ainsi au spectateur de conclure son expérience de visionnage sur le Michael Jackson performeur d'exception ainsi que sur une note renforcée de liveness, dotant du même coup le film d'un fort potentiel d'authentification (231).

Tous ces chapitres déploient une panoplie de méthodes intéressantes, dont l'analyse musicale est le socle, dans un jeu bienvenu avec les analyses d'images et les analyses plus culturelles. Considérations sur la soundbox (Moore et Dockwray), spectrogrammes (qui demanderaient à être affinés parfois), tableaux comparatifs de toutes sortes entre les diverses versions de chansons et de morceaux, analyse mélodique, plus ponctuellement : tout cela complète et étaye la saisie et la compréhension des enregistrements musicaux ou audiovisuels du corpus. Une telle abondance de constats et de résultats précis rend possibles les montées en généralité théorique dont l'auteur est friand : il échappe ainsi à la simple suite d'études de cas que son plan, les redondances qu'il induit et les défauts de limpidité résultants auraient pu produire.

Une autre qualité - encore trop rarement combinée à la saisie musicologique est omniprésente dans l'ouvrage : il offre un discours sociologique non naïf sur ses objets. La manière dont le prestige culturel et musical s'établit de manière réciproque dans la série des American, entre Johnny Cash et Rick Rubin d'un côté (le musicien
« repreneur » et son producteur) et de l'autre côté les artistes repris, est ainsi mise en évidence sans platitude cultuelle; l'ironie des carrières posthumes est soulignée, sans aller jusqu'à la caricature; la mise en sens des appareillages techniques sonores est également prise avec une juste distance critique - contrairement aux simplistes équations sémantiques trop fréquemment proposées en popular music studies.

Au terme de la lecture, entre stimulation intellectuelle et indigestion, restent deux questions en suspens. À vrai dire, elles se posent à une échelle bien plus large que celle de ce seul ouvrage : en ne les approfondissant pas, Bratus ne fait rien de bien particulier. Mais elles me paraissent pertinentes en tant que suite à donner aux préoccupations générales des popular music studies. Tout d'abord il est dommage de réduire « la technologie » aux « machines » et lesdites machines à un ensemble d'appareils électroniques et informatiques, toujours les mêmes et énumérés en une série presque fermée : aux équipements de studios d'enregistrement, on ajoute classiquement les platines à vinyles, synthétiseurs, boîtes à rythmes, sampleurs, ordinateurs et logiciels musicaux. Une telle manière de (ne pas) penser ce qu'est « la » technologie permet de trop faciles antithèses entre « vrais » musiciens et machines (une antithèse qui affleure beaucoup dans le chapitre sur l'EDM), ainsi qu'entre les genres musicaux « centrés sur la technologie » (le hip-hop, l'EDM) et les autres. L'auteur verse tellement peu dans l'approximation par ailleurs, qu'on est étonné de retrouver celle-ci dans son texte. Les instruments de musique - y compris acoustiques - sont bien évidemment des appareils relevant de technologies complexes. Un vrai questionnement de ce concept de technologie 
en musique produirait des résultats et des débats tout à fait passionnants, aussi passionnants que ceux sur le concept de liveness tels qu'Auslander en avait posé les termes en I999.

Enfin, on ne m'en voudra pas de remarquer que le corpus de l'ouvrage ne contient aucune femme. Il est très possible que les carrières musicales de femmes, avec tous les biais qu'on leur connaît, très bien étudiés maintenant, ne débouchent pas sur une phase posthume comme celles des hommes considérés ici. Toutefois, de la part d'un auteur qui lit et cite si généreusement des auteures et qui maîtrise avec tant d'aisance beaucoup de problématiques d'actualité en popular music studies, on pouvait espérer trouver, serait-ce au détour d'un de ses si nombreux développements centrifuges, des considérations tout à fait pertinentes sur cette regrettable disparité.

Mais ces deux remarques sont en réalité des critiques légères au regard de la somme réflexive proposée ici par Bratus. Ce livre est aussi dense que son titre et fournit aliment à la pensée pour longtemps. Il balaye un territoire très vaste. Il est plein de reliefs, le moindre n'étant pas de choisir comme clé centrale d'analyse une notion - le will to believe - issue de la pensée d'un psychologue lointain, William James : cela paraît un peu décalé - comme s'il reprenait un concept à Freud ou à Jung - et encore ceux-ci sont-ils plus récents que James. Après ce voyage sans guère de plan ni de boussole dans une prose analy tique et théorique touffue mais souvent passionnante, on peut choisir de croire à ce que le frère de William James, Henry, appelait le "motif dans le tapis » (the figure in the carpet) : la cohérence d'une pensée au-delà du fourmillement parfois

\section{Thomas Horeau,}

Le jazz et la scène, Saint-Denis, Presses universitaires de Vincennes, coll. "Théâtres du monde ", 2019

\section{Par Clément Bresch}

D'une certaine manière, on peut appréhender l'ouvrage de Thomas Horeau comme une saisie au pied de la lettre de la constatation quelque peu rudimentaire selon laquelle lorsque l'on se rend à un concert de jazz, on va y voir des musiciens jouer. Une saisie qui s'avère, du fait de l'éclairage original de la pratique jazzistique qu'elle engendre, tout à fait féconde. Car si le lieu emblématique du jazz n'est autre que la scène, si son actualisation paradigmatique demeure la performance, si son devenir-musique passe inéluctablement par le jeu, alors il y a effectivement un champ d'études, de prime abord peut-être guère connexe, qui pourrait avoir somme toute quelque chose à en dire : celui qui a fait du théâtre son objet.

Séparant dans le titre la chose du lieu - comme pour rappeler que l'une n'a pas le privilège de l'autre -, Le jasz et la scène se propose ainsi d'approcher la pratique jazzistique à l'aune d'une réflexion sur le champ scénique lui étant a priori extérieure : celle 